\title{
Determination of Concentrations of Glucose and Human Serum Albumin in Mixtures in Phosphate- Buffered Solution by Near-Infrared Spectroscopy
}

\author{
Mitsuhiro Ogawa, ${ }^{* * *}$, Takehiro Yamakoshi, Kosuke Motoi, Koji Nishida, \\ Yasuhiro Yamakoshi ${ }^{1}$ and Ken-Ichi Yamakoshi \\ Graduate School of Natural Science and Technology, Kanazawa University, \\ Kakuma, Kanazawa, Ishikawa 920-1192, Japan \\ ${ }^{1}$ yu.sys Corporation, 2-7-1 Ishibiki, Kanazawa, Ishikawa 920-0935, Japan
}

(Received November 1, 2011; accepted March 6, 2012)

Key words: blood constituent measurement, glucose, human serum albumin, near-infrared spectroscopy, reagentless measurement

Near-infrared (NIR) absorbance spectra of glucose and human serum albumin (HSA) mixtures in phosphate-buffered solution (PBS) $(\mathrm{pH}=7.4)$ were investigated as the basis of reagentless blood constituent measurements. Diffuse reflectance spectra of powdered glucose and HSA were also studied. Differential absorbance spectra taken relative to the spectrum of a reference PBS were measured in the wavelength range of 750-2,500 nm. In the differential spectra of mixture sample solutions, positive peaks corresponding to the HSA concentration could be observed, particularly in the range of 2,150-2,350 nm; however, peaks corresponding to the glucose concentration could not be observed. Some peaks visible in the diffuse reflectance spectra of powdered HSA could not be observed in the differential spectra. Measured NIR spectra were subjected to partial least-squares regression and good predictions of both HSA and glucose concentrations were obtained. Calibration for glucose in the concentration range of $0-300 \mathrm{mg} / \mathrm{dl}$ yielded a correlation coefficient of 0.9965 and a standard error of prediction (SEP) of $8.3 \mathrm{mg} / \mathrm{dl}$ with a latent variable of five. Calibration of HSA within the range of $2-4 \mathrm{~g} / \mathrm{dl}$ yielded a correlation coefficient of 0.9996 with a SEP of $0.023 \mathrm{~g} / \mathrm{dl}$ and a latent variable of five. These results suggest that reagentless measurements of glucose and HSA concentrations in human serum are possible with NIR spectroscopy.

\footnotetext{
${ }^{*}$ Corresponding author: e-mail: ogawa@his.teikyo-u.ac.jp

${ }^{* *}$ Present affiliation: Faculty of Science and Engineering, Teikyo University (1-1 Tokyosato-Dai, Utsunomiya, Tochigi 320-5551, Japan)
} 


\section{Introduction}

From a clinical viewpoint, blood substances can provide much valuable information about the physiological status of an individual. Because of this importance, many attempts to determine these substances have been reported over the past century. Although current blood substance analyses mainly involve chemical and/or biological reactions with reagents, because of the high precision of such measurement, blood constituent measurement without any reagents, also called "reagentless measurement", has been studied in tandem with the advancement of measurement instrumentation and applied statistics. $^{(1,2)}$ Reagentless monitoring allows the possibility of measurement with rapid response, real-time monitoring, cost-effectiveness, and simultaneous measurement of multiple constituents with only one device. ${ }^{(1-3)}$ Thus, there have been many attempts to realize this approach. An example of such reagentless measurement, with a long history, is CO-oximetry, that is, the in vitro measurement of the oxygenation status of hemoglobin contained in red blood cells using visible light and near-infrared (NIR) radiation. ${ }^{(4,5)}$ Furthermore, in vitro CO-oximetry has been expanded to the in vivo and noninvasive measurement technique named "pulse oximetry", which aims to measure the oxygenation status of a patient's hemoglobin transcutaneously. ${ }^{(6)}$ Attempting to add to the success of these methods for hemoglobin oxygenation measurement, the development of reagentless techniques for blood constituents, other than those linked to oxygen, is still ongoing.

In efforts to develop methods for the reagentless measurement of blood constituents, researchers continue to explore the use of optical techniques, using visible and infrared radiation, because such measurement procedures can be applied to blood, plasma, and/or serum nondestructively. Among these efforts, many promising attempts using optical absorbance measurement have been reported in recent decades. In these previous attempts, interest was mainly focused on clinically important blood constituents such as total hemoglobin, glucose, protein, and cholesterol.(1,3,7-14) From a methodological viewpoint, many recent advances in reagentless measurement have been based on the combined power of NIR spectroscopy and chemometric analysis. Chemometrics is an important field within applied chemistry, whereby chemical information is acquired by data-driven means, using advanced statistical and applied mathematical methods, and computing science. Although chemometric methods applied to quantitative NIR spectroscopy have the potential to extract small components from the original measured data, the extraction procedure is highly dependent on the data-driven means employed. Consequently, the results that emerge could sometimes be unclear or inaccurate if there are artifacts with spurious correlation.

Although there have been many reports of promising results of studies using NIR spectroscopy, most of such studies have been highly dependent on chemometrics, in which several different statistical methods have been investigated. The mechanisms and origins of the measurements are still unclear in many cases; thus, it is difficult, in practice, to compare those results and establish a standard calibration. Although many previous attempts have been focused particularly on statistical analyses to form a calibration model, we consider that the data and parameters obtained, such as absorbance 
spectra, should be discussed in more detail to establish a standard method of reagentless monitoring of blood substances. The adequacy of the results obtained from the diffuse reflectance spectra of powdered blood constituents has been reported and there was an implicit assumption that spectral peaks in the powder state could be observed in aqueous solutions, in serum and/or whole blood. ${ }^{(8)}$ However, this implicit assumption had not been justified. Previously, we attempted to observe positive peaks in the absorbance spectrum of human serum albumin (HSA) in phosphate-buffered solution (PBS) using differential spectra from a reference solution spectrum and found clear peaks corresponding to HSA concentration. ${ }^{(15)}$ We also found that some peaks that could be observed in diffuse reflectance spectra of powder-state HSA could not be observed, and limited peaks in the powder state could be apparent in aqueous solution.

Moreover, we have recently proposed a novel noninvasive method of in vivo measurement of blood glucose concentration using an NIR multiwavelength photoplethysmogram (PPG), which was named pulse glucometry. ${ }^{(16-18)}$ The measurement principle of pulse glucometry is based on the fact that the glucose molecule has absorption bands in the NIR region and the PPG is derived from the arterial volume change of the blood vessel at the measurement site (e.g., fingertip). In vitro optical reagentless measurement can be an important basis of the further development of this in vivo pulse glucometry technique.

In this study, we tried to expand on our previous attempt and endeavored to observe the spectral features of glucose and HSA mixture solutions, and then form a calibration model for the determination of glucose and HSA concentrations in a solution.

\section{Materials and Methods}

\subsection{Sample preparation}

Glucose powder $(\mathrm{D}(+)$-glucose, 043-31163, Wako Pure Chemical Industries Ltd., Japan) and HSA (019-10503, Wako Pure Chemical Industries Ltd., Japan) were used in the experiments. The purity of the HSA sample was proved to be greater than $95 \%$ by electrophoresis.

Two groups of glucose and HSA samples were prepared. Glucose powder and HSA samples were used for group I. Group II consisted of glucose and HSA powders in PBS. PBS was prepared using purchased PBS powder (167-14491, Wako Pure Chemical Industries Ltd., Japan) and pure water supplied from an ultrapure water production system (Autopure WT100, Yamato Corp., Japan). After sterilizing PBS, its pH was adjusted to 7.4, a typical $\mathrm{pH}$ of blood, serum, and plasma in a healthy human. The concentrations of the prepared glucose and HSA in solution samples were in the range of 0 to $300 \mathrm{mg} / \mathrm{dl}$ and 2.0 to $4.0 \mathrm{~g} / \mathrm{dl}$, respectively, as shown in Table 1 . The concentration ranges of glucose and HSA cover the concentrations in the blood of a healthy human and a diabetic patient. In addition to mixture solutions, pure glucose solutions of 100 to $500 \mathrm{mg} / \mathrm{dl}$ were prepared. To prepare sample solutions, glucose powder and HSA were weighed using an electronic balance and the volumes of solutions were made up using volumetric flasks. 
Table 1

Concentrations of glucose and human serum albumin in samples.

\begin{tabular}{cc}
\hline & Concentration \\
\hline Albumin $(\mathrm{g} / \mathrm{dl})$ & Glucose $(\mathrm{mg} / \mathrm{dl})$ \\
\hline 2 & $0,50,100,150,200,250,300$ \\
3 & $0,50,100,150,200,250,300$ \\
4 & $0,50,100,150,200,250,300$ \\
\hline
\end{tabular}

Albumin: human serum albumin

\subsection{Instruments}

A Fourier transform near-infrared (FT-NIR) spectrometer (Spectrum One NTS, PerkinElmer Japan Co., Ltd, Japan) was used for absorbance measurements. The measured wavelength range was $750-2,500 \mathrm{~nm}$ and the resolution was $1.66 \mathrm{~nm}$. For group I, diffuse reflectance absorption spectra of glucose and HAS powder were measured using a diffuse reflectance cell. For group II, a flow cell of $1 \mathrm{~mm}$ optical path length with quartz plate windows was used for measurement in the NIR region (Quartz Glass Products, 6220-72011, GL Sciences Inc., Japan). For each sample in group II, absolute absorbance spectra (background measurement was obtained using no sample) of samples and differential absorbance spectra relative to the spectrum of a reference PBS ( $\mathrm{pH} 7.4$; concentrations of glucose and HSA, $0.0 \mathrm{~g} / \mathrm{dl}$ each) were measured. The room temperature and solution sample temperatures were both kept at $27.0 \pm 0.3^{\circ} \mathrm{C}$ during the measurements. In group II, the sequence of measurements was random.

\subsection{Data processing and calibration model}

The software "R" (version 2.12.2), its extension package "pls" (version 1.2-1), and "RTisean" (version 3.0.11) were used for spectral data processing and forming the calibration model. ${ }^{(19)}$ All data from group II were processed using the SavitzkyGolay filter to eliminate noise and artifacts by conventional chemometry. Multivariate regression analysis of group II samples was then performed to form a calibration model for estimating both glucose and HSA concentrations. In this study, a classical partial least-squares (PLS) regression was applied to the spectral data after Savitzky-Golay filtering to analyze data and form calibration models.

\section{Results}

\subsection{NIR spectra of glucose and HSA powders}

The measurements of glucose and HSA powder samples (group I) using the FTNIR spectrometer provided NIR diffuse-reflectance absorption spectra. Figure 1 shows diffuse-reflectance absorption spectra of glucose and HSA powders in the 750-2,500 $\mathrm{nm}$ range. In these spectra, several positive (convex) peaks can be observed for both samples. The difference between the forms of the spectra of glucose and HSA is clearly observed in Fig. 1. As was suggested in a previous paper, ${ }^{(3)}$ the spectral peaks contain information about the molecular features of glucose and HSA. 


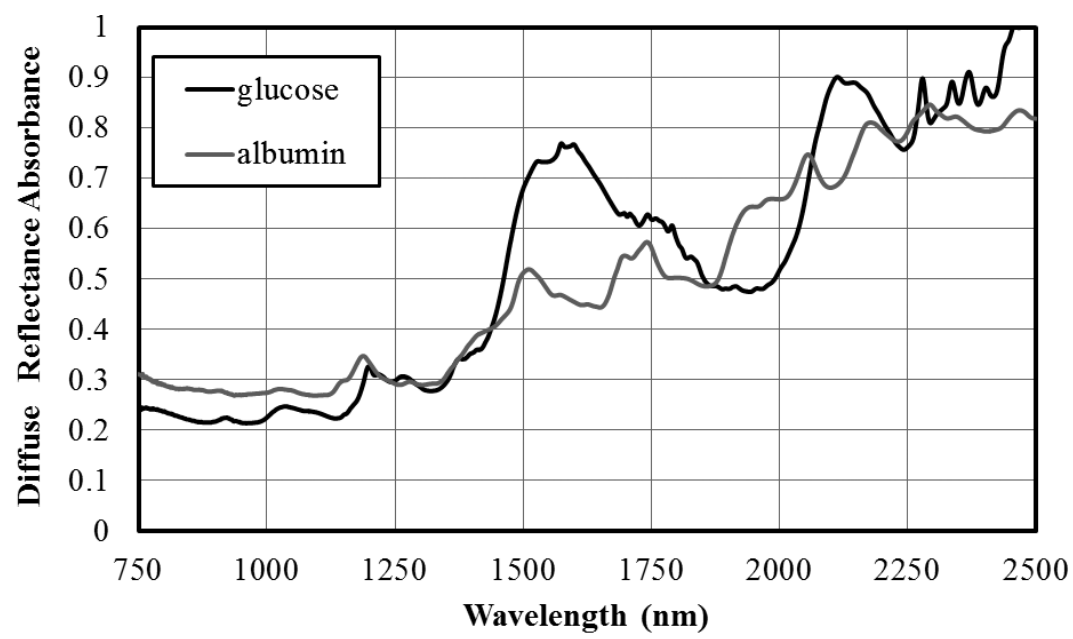

Fig. 1. NIR diffuse-reflectance spectra of glucose and human serum albumin powders.

\subsection{Absolute absorption of samples}

Absolute absorption spectra of glucose and HSA mixture solutions were measured in the 750-2,550 nm range. The forms of the spectra were similar to our previous results for absolute absorption spectra of HSA sample solutions. ${ }^{(14)}$ In the obtained spectra, strong absorption peaks derived from water $\left(\mathrm{H}_{2} \mathrm{O}\right)$ absorption ${ }^{(20,21)}$ were observed in the $1,450-1,900 \mathrm{~nm}$ range. Because such strong water absorption completely masks the absorption of glucose and HSA, it is very difficult to observe any features of dissolved glucose and HSA in the absolute absorption spectra.

\subsection{Differential absorption spectra of glucose and HSA solutions from the spectrum of reference solution}

Examples of differential absorption spectra of glucose and HSA solution samples relative to a reference PBS spectrum in the 750-2,550 nm range are shown in Figs. 2 and 3. Figure 2 shows differential absorption spectra of pure glucose solutions. Figure 3 shows differential absorption spectra of glucose and HSA mixture solutions. The baseline shift on the measured spectra was removed by subtracting the absorbance at $1,200 \mathrm{~nm}$. As shown in Figs. 2 and 3, the band near 1,950 nm and over 2,400 nm cannot be measured because of the strong water absorption in the band. The observed negative (concave) peak at $1,450 \mathrm{~nm}$ is also considered to be caused by a change in the amount of water in the sample. This is because samples with higher glucose and HSA concentrations contain smaller amounts of water per unit volume. Consequently, samples with higher glucose and HSA concentrations must have smaller absorptions in the water-dominant 


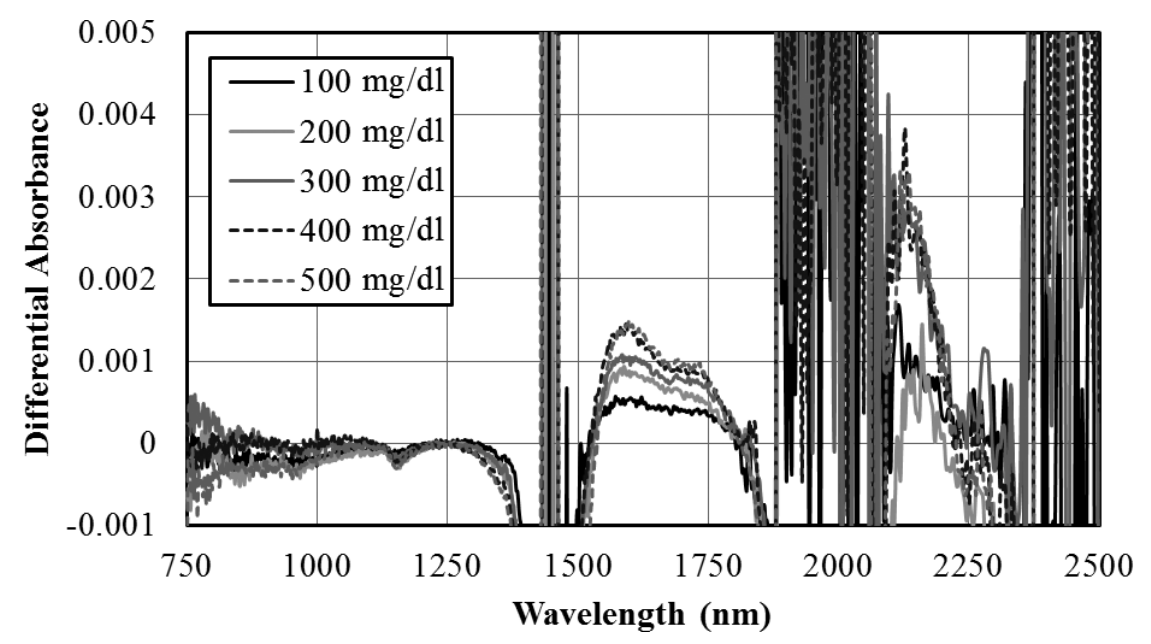

Fig. 2. Examples of NIR differential absorbance spectra of pure glucose in PBS. Differential absorbances were measured against the reference PBS.
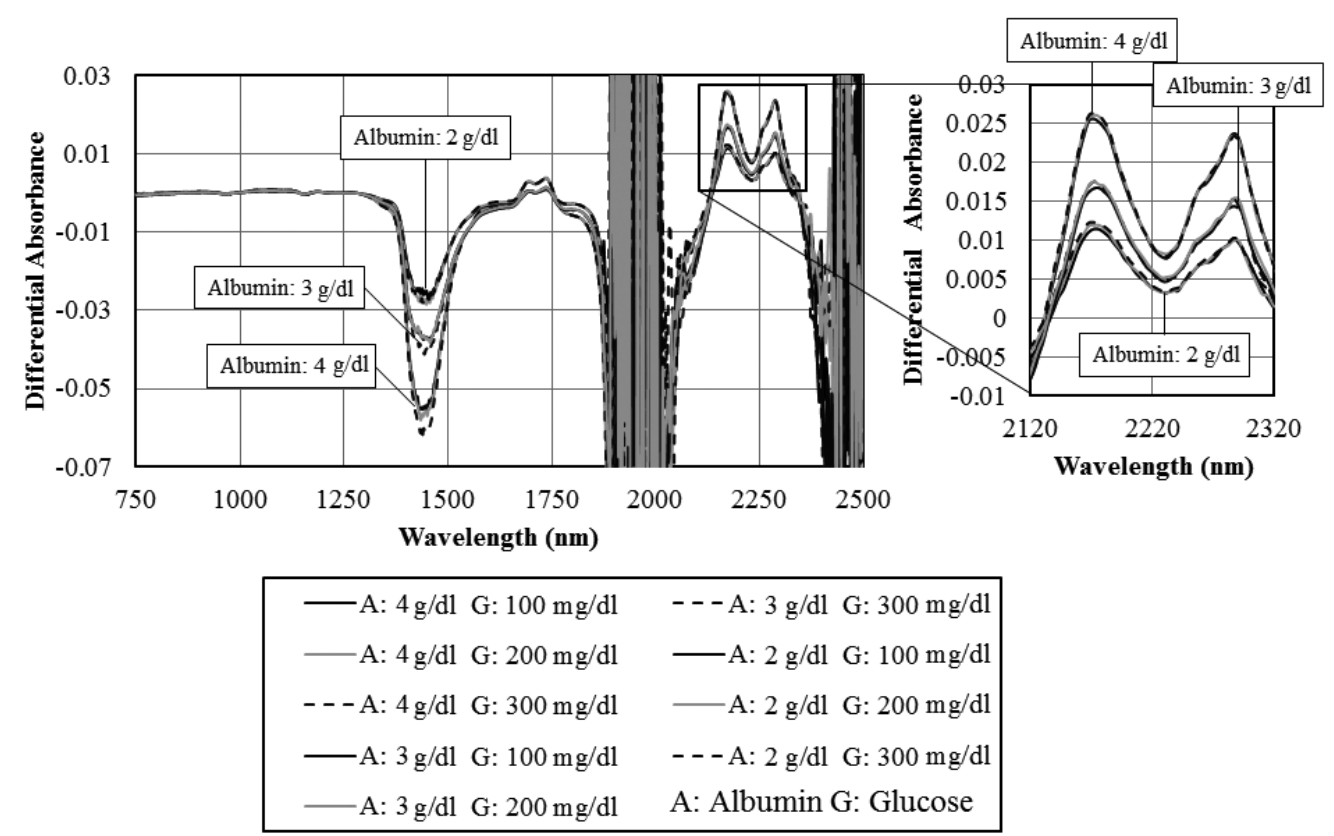

Fig. 3. Examples of near-infrared differential absorbance spectra of glucose and human serum albumin mixtures in PBS. Differential absorbances were measured against the reference PBS. 
absorption regions such as near $1,450 \mathrm{~nm}$. In contrast, a positive peak that corresponds to glucose concentration can be observed in the 1,600-1,700 nm range in Fig. 2. In Fig. 3, the clearly observable peaks in the $1,650-1,750 \mathrm{~nm}$ and $2,150-2,350 \mathrm{~nm}$ ranges should be considered as corresponding to the HSA concentration of the samples, as in our previous results. ${ }^{(15)}$ Additionally, the position of the positive peaks coincided well with the position of the convex peaks on the diffuse reflectance spectra of the glucose and HSA powders. However, limited peaks for the powder state could be found in aqueous solution as found in our previous study. ${ }^{(15)}$ Convex peaks at approximately 1,600 and 2,100 $\mathrm{nm}$ can be observed in both Figs. 1 and 2. The peak at approximately 1,200 in Fig. 1 cannot be found in Fig. 2. The dependence of the positive peak on glucose concentration cannot be observed from the absorbance spectra of glucose and HSA mixture solutions, because the NIR absorbance of glucose is weaker than that of HSA at the selected concentration.

\subsection{Calibration models for determination of glucose and HSA concentrations}

Classical PLS regression analysis was adopted to correlate the NIR differential spectra from samples with various glucose and HSA concentrations. First, the determination of HSA concentration was attempted, because HSA has more intense absorption peaks than glucose in our samples. The differential absorbance spectra at the 1,650-1,750 nm and $2,250-2,250 \mathrm{~nm}$ ranges were selected as explanatory variables and HSA concentration as the dependent variable for PLS analysis. Bands other than the observed positive peaks were rejected from the analysis in advance. Differential absorbances of 122 wavelengths were examined, and the leave-one-out cross-validation technique was applied to evaluate the regression. As a result, the latent variable five in the PLS analysis was obtained as the optimum parameter, and the regression provided a standard error of prediction (SEP) of $0.023 \mathrm{~g} / \mathrm{dl}$. Figure 4 shows the results of the leave-one-out cross-validation and the correlation coefficient between the actual HSA concentration and predicted concentration is 0.9996 . This result shows that HSA concentrations in the range expected in healthy humans can be estimated, in the presence of glucose, on the basis of the selected bands.

Second, the determination of glucose concentration was attempted. The differential absorbance spectra for the 1,550-1,750 nm range were selected for PLS regression. Differential absorbances of 122 wavelengths were examined as explanatory variables. The leave-one-out cross-validation technique was also applied, and the optimum latent variable five for PLS yielded the regression with SEP of $8.3 \mathrm{mg} / \mathrm{dl}$. Figure 5 shows the results of the leave-one-out cross-validation, with a correlation coefficient of 0.9965 . This result shows that glucose concentrations in the range expected for human subjects can be estimated in the presence of an interfering substance, HSA.

\section{Discussion}

Concerning the comparison of diffuse reflectance spectra of glucose powder with differential spectra of glucose solution, limited peaks of glucose powder were only apparent in glucose solution, as in our previous result for HSA. ${ }^{(15)}$ This finding suggests the importance of an accurate absorbance measurement by differential spectral 

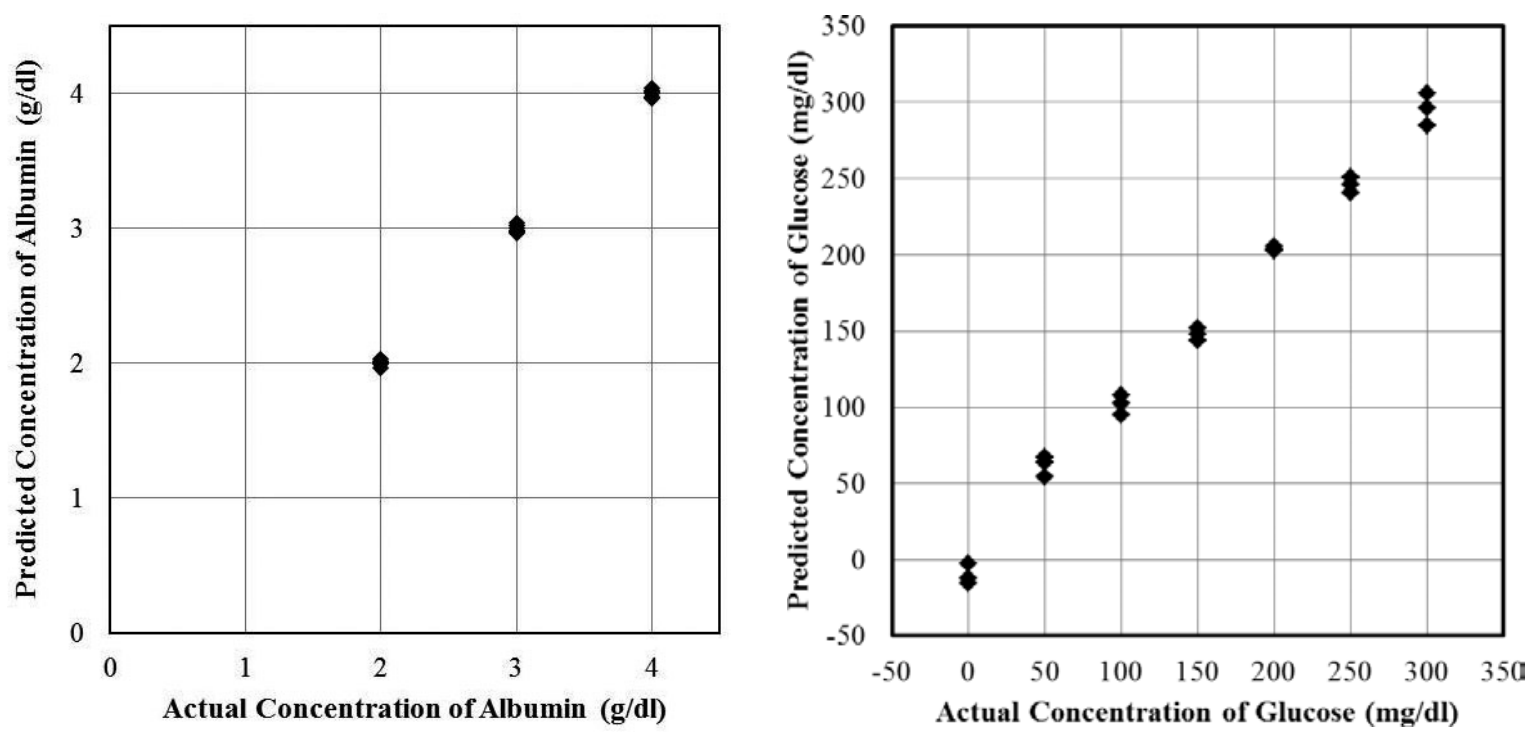

Fig. 4 (left). Calibration plot of estimated concentration of human serum albumin in mixtures of solutions against actual concentration based on leave-one-out cross-validation.

Fig. 5 (right). Calibration plot of estimated concentration of glucose in mixtures of solutions against actual concentration based on leave-one-out cross-validation.

observation.

A recently proposed in vivo blood glucose measurement, pulse glucometry, is based on an NIR multiwavelength PPG that can contain information on arterial blood constituents. We previously attempted PPG spectral analysis using PLS regression, principal component regression, and also support vector machine regression, ${ }^{(17,18)}$ and obtained good estimates of blood glucose concentrations. However, current pulse glucometry is dependent on multivariate analyses using multiwavelength PPG with about 100 wavelengths. Our final goal is to develop a new cost-effective and noninvasive self-monitoring blood glucose (SMBG) device based on pulse glucometry to replace the current finger-pricking SMBG meters and to expand it to other blood chemical measurements. To achieve our final goal, neither a Fourier transform infrared (FT-IR) spectrometer nor a diffraction-grating spectrometer can be used. One possibility for actualization would be spectrophotometry using multiple light-emitting diodes (LEDs) as light sources. In this case, an optical measurement of many wavelengths with very narrow wavelength resolution, as in an FT-IR or diffraction-grating spectrometer, cannot be expected. Therefore, we tried to form calibration models using a smaller number of wavelengths with a wider wavelength resolution. Measured differential absorbance was averaged within a predefined range and adopted as an explanatory variable in PLS regression. This should simulate spectrometry using one or more LEDs. For 
determination of HSA concentration, differential absorbance was selected and averaged within each of the ranges 1,650-1,700, 1,701-1,750, 2,150-2,200, and 2,201-2,250 nm, and used as four explanatory variables. For determination of glucose concentration, differential absorbance within each of the ranges 1,550-1,600, 1,601-1,650, 1,651-1,700, and 1,701-1,750 nm was selected, averaged, and again adopted as four explanatory variables. As a result of the HSA concentration determination, the optimum latent variable of four was obtained and the PLS regression provided an SEP of $0.0181 \mathrm{~g} / \mathrm{dl}$. Figure 6 shows the results of the leave-one-out cross-validation, and the correlation coefficient was 0.9998 . In the determination of glucose concentration, the optimum latent variable of four was obtained, and the PLS regression provided an SEP of $12.3 \mathrm{mg} /$ dl. Figure 7 shows the results of the leave-one-out cross-validation, with a correlation coefficient of 0.9924 . This result can form the basis of the design of an LED-based spectroscopy system for in vivo blood glucose and HSA concentration measurement.

Li et al. conducted in vitro and in vivo blood glucose measurements by NIR spectroscopy, and when combined with their sophisticated data-driven chemometrics procedure, they found good results. ${ }^{(14)}$ They selected informative spectral regions for
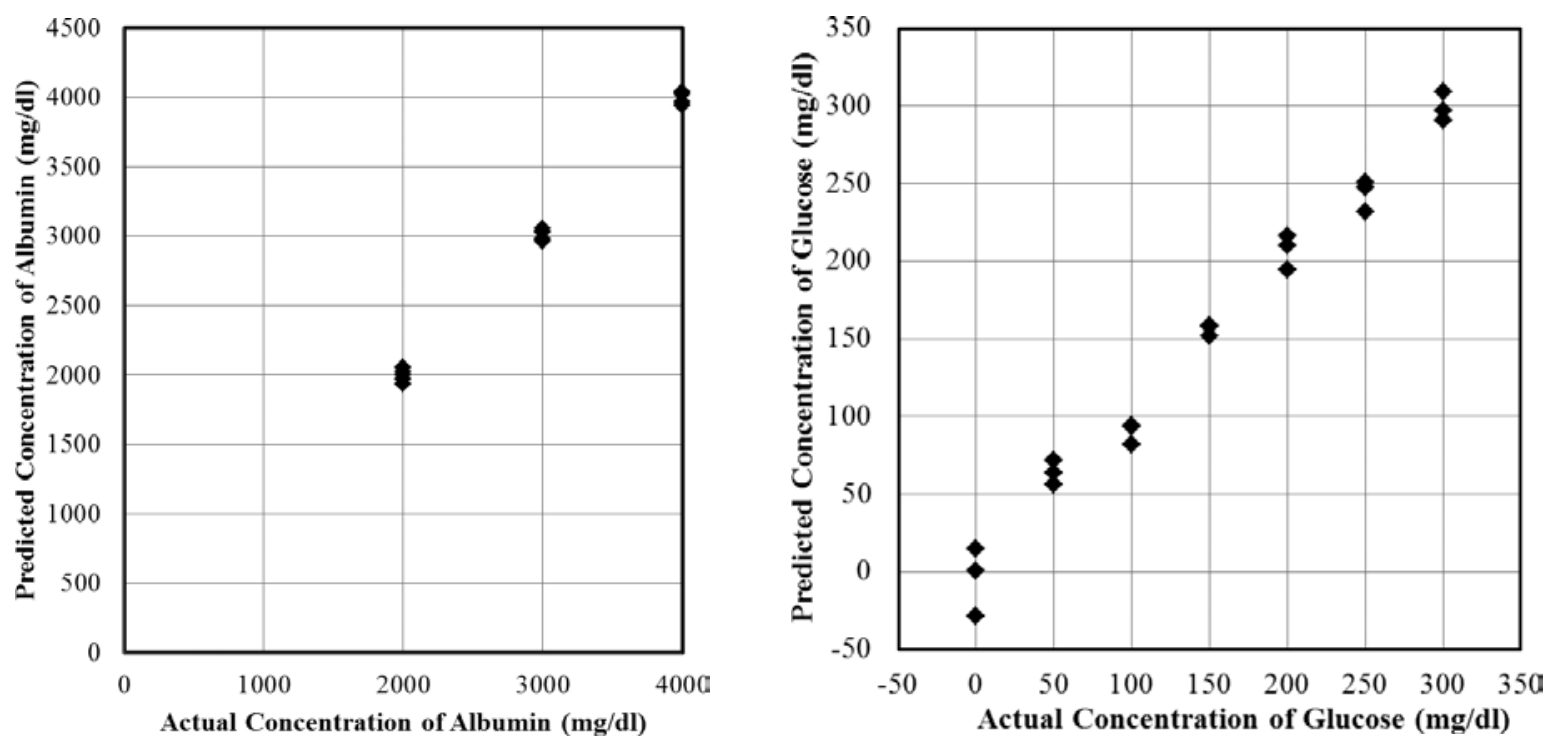

Fig. 6 (left). Calibration plot of estimated concentration of human serum albumin in mixtures of solutions against actual concentration using averaged absorbances based on leave-one-out crossvalidation.

Fig. 7 (right). Calibration plot of estimated concentration of glucose in mixtures of solutions against actual concentration using averaged absorbances based on leave-one-out cross-validation. 
prediction of HSA and glucose concentrations by their statistical method. Their reported informative ranges were 5,907-5,663 $\mathrm{cm}^{-1}(1,692-1,765 \mathrm{~nm}$ wavelength) and 4,648$4,323 \mathrm{~cm}^{-1}(2,151-2,313 \mathrm{~nm})$ for HSA, and those ranges coincide well with our results. In addition, their reported informative ranges of glucose were $6,080-5,700 \mathrm{~cm}^{-1}(1,664$ $1,754 \mathrm{~nm}$ in wavelength) and 4,647-4,255 $\mathrm{cm}^{-1}(2,152-2,350 \mathrm{~nm})$ and the former range falls within our selected range for glucose prediction. Although our methodology was completely different in principle from that of $\mathrm{Li}$ et al., approximately the same results could be obtained. This can possibly support the adequacy of our results and the results of Li et al.

Although we discussed a simple HSA solution previously ${ }^{(15)}$ and a glucose and HSA mixture solution in this paper, real blood, serum, and/or plasma are more complicated and composed of many substances. In the next step, we plan to measure and examine control plasma and real blood samples, then attempt to form a calibration model using a small number of absorbances with a wider wavelength resolution.

\section{Conclusions}

NIR absorbance spectra of glucose and HSA mixture sample solutions were discussed as the basis of reagentless blood constituent measurement. Differential absorbance spectra from the spectrum of a reference PBS were measured in the wavelength range of 750-2,500 nm. In the differential spectra of mixture sample solutions taken relative to the reference spectrum, positive peaks corresponding to HSA concentration could be observed, particularly in the 1,650-1,750 and 2,150-2,350 nm ranges; however, peaks corresponding to glucose concentration could not be observed. NIR spectra were subjected to partial least-squares regression, and good predictions of the HSA and glucose concentrations were obtained. These results are considered as the basis of reagentless measurement of HSA and glucose concenstrations in human serum as well as in vivo glucose and HSA concentration measurement.

\section{Acknowledgements}

This research was partially supported by the Ministry of Education, Culture, Sports, Science and Technology, Japan through Grant-in-Aid for Scientific Research (A), 21240047, 2009-2011. The authors are grateful for the support.

\section{References}

1 S. Hahn, G. Yoon, G. Kim and S. Park: J. Opt. Soc. Korea 7 (2003) 240.

2 M. Mejri, H. Baccar, D. Baldrich, F. Del Campo, S. Helali, T. Ktari, A. Simonian, M. Aouni and A. Abdelghani: Biosens. Bioelectron. 26 (2010) 1261.

3 K. Murayama, K. Yamada, R. Tsenkova, Y. Wang and Y. Ozaki: Vib. Spectrosc. 18 (1998) 33.

4 J. Severinghaus and P. Astrup: J. Clin. Monit. Comput. 2 (1986) 1387.

5 D. Drabkin and J. Austin: J. Biol. Chem. 112 (1935) 105.

6 T. Aoyagi and K. Miyasaka: Anesth. Analg. 94 (2003) S1.

7 G. Farace, G. Lillie, T. Hianik, P. Payne and P. Vadgama: Bioelectrochemistry 55 (2002) 1. 
8 S. Kasemsumran, Y. Du, K. Murayama, M. Huehne and Y. Ozaki: Analyst 128 (2003) 1471.

9 J. Hall and A. Pollard: Clin. Biochem. 26 (1993) 483.

10 D. Haaland, M. Robinson, G. Koepp, E. Thomas and R. Eaton: Appl. Spectrosc. 46 (1992) 1447.

11 J. Hall and A. Pollard: Clin. Chem. 38 (1992) 1623.

12 A. van Toorenenbergen, B. Blijenberg and B. Leijnse: Clin. Chem. Lab. Med. 26 (1998) 209.

13 E. Peuchant, C. Salles and R. Jensen: Anal. Chem. 59 (1987) 1816.

14 B. Y. Li, S. Kasemsumran, Y. Hu, Y. Z. Liang and Y. Ozaki: Anal. Bioanal. Chem. 387 (2006) 603.

15 M. Ogawa, Y. Yamakoshi, T. Yamakosi and K. Yamakoshi: Internet J. Bioeng. 4 (2010) http://www.ispub.com/journal/the_internet_journal_of_bioengineering.html (accessed on 5 February 2012).

16 K. Yamakoshi: International Patent No. PCT/JP03/03587 (2003).

17 K. Yamakoshi and Y. Yamakoshi: J. Biomed. Opt. 11 (2006) 054028.

18 Y. Yamakoshi, M. Ogawa and T. Tamura: Open Opt. J. 3 (2009) 63.

19 R Development Core Team, R: A Language and Environment for Statistical Computing (R Foundation for Statistical Computing, Vienna, 2005).

20 F. Palmer and D. Williams: J. Opt. Soc. Am. 64 (1974) 1107.

21 J. Wang, R. Xu and S. Yang: Environ. Monit. Assess. 157 (2009) 459. 